\title{
LA INFLACIÓN EN LA PERSPECTIVA DE LA ESCUELA AUSTRIACA
}

\author{
Oshiel Martínez Chapa* \\ https://orcid.org/0000-0001-9675-5472
}

RECIBIDO: Enero 2020 / ACEPTADO: Febrero 2020 / PUBLICADO: Mayo 2020

Como citar: Martínez Chapa, Oshiel (2020). La inflación en la perspectiva de la escuela austriaca. Telos: revista de Estudios Interdisciplinarios en Ciencias Sociales, 22 (2), Venezuela. (Pp.325341).

DOI: www.doi.org/10.36390/telos222.06

\section{RESUMEN}

El presente trabajo analiza el problema de la inflación desde la perspectiva de la Escuela Austriaca (EA), pues aun cuando se le conceptúe como "antigua", pues data de finales del siglo $\mathrm{XIX}$ y principios del XX, tiene una explicación respecto a este y otros problemas macroeconómicos. Destacan autores como. Este paradigma se enriqueció con los aportes de Mises (1934) con Teoría del Dinero y el Crédito, Hayek (1985), con La Desnacionalización del Dinero, Ropke (1960),, con A humane Economy y Rothbard (1990) con Teoría austriaca del dinero. Sin embargo, también se hace alusión respecto a esta problemática por parte de los autores de la denominada corriente principal. Se recurre a información de tipo cualitativo y cuantitativo. Como resultado de este trabajo se señala que, aunque las demás corrientes de pensamiento económico no difieren mucho en señalar los efectos de la inflación para la sociedad, la Escuela Austriaca, identifica como causas a la expansión crediticia y al déficit y deuda pública creciente. Como hallazgos derivados de la revisión de la literatura deben señalarse al creciente intervencionismo estatal, fuertes expansiones en el crédito, distorsiones en los precios y la presencia de ciclos de expansión y contracción en la economía como regularidad. De esto modo, se concluye que las instituciones financieras y políticas deben velar por el crecimiento económico con estabilidad por el bien de la sociedad.

Palabras clave: Inflación, ciclo económico, políticas de estabilización, Escuela Austriaca.

\section{Inflation in Austrian School perspective}

\begin{abstract}
This paper analyzes the problem of inflation from the perspective of the Austrian School (EA), because even if it is conceptualized as "old", since it dates from the late 19th and early 20th centuries, it has an explanation regarding this and Other macroeconomic problems Authors stand out as. This paradigm was enriched with the contributions of Mises (1934), with Theory of Money
\end{abstract}

\footnotetext{
* Doctor en Economía por el Instituto Internacional de Derecho y del Estado, Chihuahua, México. Profesor de la Universidad Autónoma de Tamaulipas y del Tecnológico Nacional de México, Reynosa, Tamaulipas, México. Correo electrónico: omartinez@uat.edu.mx
} 
and Credit, Hayek (1985), with The Denationalization of Money, Ropke (1960), with A Humane Economy and Rothbard (1990), with Austrian Theory of Money. However, reference is also made regarding this issue to the position of this problem by the authors of the so-called mainstream. Qualitative and quantitative information is used. As a result of this work it is pointed out that, although the other currents of economic thought do not differ much in pointing out the effects of inflation for society, the Austrian School identifies as causes the credit expansion and the deficit and increasing public debt. As findings derived from the review of the literature should be noted the growing state intervention, strong expansions in credit, distortions in prices and the presence of cycles of expansion and contraction in the economy as a regularity. In this way, it is concluded that financial and political institutions must ensure economic growth with stability for the good of society.

Keywords: Inflation, economic cycle, stabilization policies, Austrian School.

\section{Introducción}

La inflación es un tema que se examina como parte de los contenidos de los cursos e investigaciones en materias de ciencia económica a nivel universitario. Sin embargo, dicho fenómeno macroeconómico no ha perdido relevancia aun cuando hayan pasado décadas de observación y de medición. El presente trabajo se justifica en tanto que la Escuela Austriaca (EA) tiene una interpretación respecto de la inflación —y de otros problemas económicos - la cual es distinta del que plantea la corriente dominante o mainstream; la misma que agrupa las visiones de las escuelas neoclásica, keynesiana y marxista, por mencionar las principales.

El presente trabajo da cuenta de diversas experiencias inflacionarias de países latinoamericanos (México, Brasil y Argentina), además de Estados Unidos, los cuales sufrieron elevadas tasas en este indicador entre los 80 s y 90 s. La hipótesis que se plantea es que existen factores impulsores de los precios, derivados estos de la estructura económica y caracterizados por la expansión del crédito, los incrementos en la oferta de dinero sin respaldo y de demanda agregada no sostenible, y que estos, eventualmente, propician la disminución de la producción, el incremento en las tasas de desempleo y mayores de niveles de endeudamiento público y privado.

Los planteamientos de la Escuela Austriaca recogen anhelos como el de la libertad económica, pero también se muestran contrarios al crecimiento del Estado como lo proponen el keynesianismo, el marxismo y el absolutismo. Sus críticas también se hacen respecto a la utilización de modelos en algunos casos poco realistas respecto al funcionamiento de la economía, así como al fracaso intelectual de la corriente principal al no anticipar las fluctuaciones que han traído graves problemas asociados a la emisión de dinero (Mises, 1934; Rothbard, 1990; Cachanosky, 1989; Huerta de Soto, 2009; Hayek, 1996).

La presente investigación tuvo como objetivo analizar el problema de la inflación y en este se emplea, aunque no de forma exclusiva una interpretación de la Escuela Austriaca (EA). El fenómeno referido es relevante hasta ahora y se aborda desde diferentes posturas científicas. En las misma se echa de la información cualitativa y analiza datos cuantitativos. El trabajo comprende las siguientes partes: Introducción; 1. La inflación según la Escuela Austriaca; 2. La inflación según las diversas escuelas de pensamiento económico contemporáneo; 3 . Efectos 
inflacionarios sobre los agentes económicos; 4. Políticas de estabilización; 5 . Conclusiones y Referencias.

\section{La inflación según la escuela austriaca: pasado y presente}

De entrada, es conveniente partir de una definición de la inflación según el paradigma de la corriente principal o mainstream, la cual en esencia se entiende como el crecimiento constante del nivel general de precios. Se trata de una cantidad de dinero considerable emitida sin respaldo, expresada en los precios de los bienes finales y cuyo proceso puede crecer indefinidamente. Debe precisarse que algunos precios tienen incrementos modestos y otros no experimentan tales incrementos. No obstante, en el ámbito académico se entiende como el cambio porcentual en el nivel general de precios (Samuelson y Nordahus, 2010 y Blanchard, 2017).

Hasta el presente continúa escribiéndose sobre este tema y se sigue insistiendo en las causas y problemas que derivan del incremento de los precios. A continuación, se señalarán algunas conceptualizaciones sobre la inflación según autores identificados con el paradigma de la EA.

El trabajo de Perdomo Ruiz (2017), parte de la definición de inflación propia de la EA, fundamentalmente basada en Rothbard (1990), según la cual dicha variable no es el resultado del nivel de precios, sino más bien de la expansión de la oferta monetaria, que excede a la demanda agregada. El autor analiza las implicaciones de la política monetaria para Colombia en el periodo 1991-2011 en el que se compara la inflación oficial del Banco Central respecto de aquella que, en teoría, se derivaría de la banca libre. Perdomo Ruiz (2017), encuentra diferencias importantes en las mediciones e interpreta los efectos que tiene el denominado ciclo económico. Este ciclo, de acuerdo con Huerta de Soto (2009), es el proceso en el que la economía toma la tasa de interés considerando solo las preferencias de los individuos y así elimina el sesgo de la expansión del crédito al que recurren los bancos centrales.

Otro trabajo que debe citarse es el de Borgucci (2011), en el que se revisan algunos aportes de la EA —en específico los de Ludwig von Mises y Friedrich Hayek — con relación a la existencia de los ciclos económicos en Venezuela en el siglo XX. El referido autor señala que dicho país se deterioró económica y socialmente como resultado de políticas como: intervencionismo; fuerte expansión del crédito; bajo nivel de inversión y de creación de empresas; distorsiones en los precios e incentivos a la producción; incremento en las importaciones y afectación en el tipo de cambio. En suma: de las recetas de la nueva macroeconomía clásica y del keynesianismo.

En esta línea de pensamiento, Adam Smith (1983), advirtió como parte del problema de la elevación de precios a la avaricia y a la injusticia de los príncipes de los Estados soberanos de su época, quienes, al abusar de la confianza de sus súbditos, disminuyeron gradualmente la cantidad verdadera del metal que primitivamente contenían sus monedas. De lo que se infiere es que los grandes capitalistas y monopolistas se enseñorean en posiciones de ventaja para incrementar sus precios según consideren maximizar sus rentas y sin que se presenten obstáculos para ello.

Las guerras tienen una implicación monetaria respecto al futuro. De hecho, el periodo de la Posguerra en los países occidentales en la mitad del siglo XX, se caracterizó por elevadas tasas de inflación, aun cuando se experimentaba una expansión económica. Sin embargo, dicho 
crecimiento se asoció al déficit y al elevado nivel de endeudamiento provocado por factores como el financiamiento de las intervenciones militares, las devaluaciones monetarias y otras formas de manipulación gubernamental. Harry Johnson (1985), lo planteó en los siguientes términos:

[...] la ola inflacionista del periodo inmediatamente posterior a la guerra (...) [fue] más severa de lo que debía haber sido. Esta particular ola inflacionista se vio agravada por las economías europeas, por la serie paralela de las devaluaciones monetarias de 1949, las cuales resultaron, a la luz de los hechos ocurridos, ser excesivas y contribuyeron a crear los serios problemas de desequilibrio monetario internacional con los que se vieron plagados los países capitalistas desde finales de los cincuenta (p.24).

En este mismo sentido, se manifiesta el economista austriaco Friedrich Hayek (1985), pues en La desnacionalización del dinero cuestiona el rol de los Estados como impulsores de tasas elevadas de inflación. Señala que las facultades de emisión y regulación de dinero no han contribuido a proporcionar monedas estables, sino que se han convertido en un instrumento de las políticas de fuerte impulso al gasto público con las consecuencias de un gobierno grande e ineficiente.

Por su parte, David Hume destacó en 1750 los efectos nocivos de la inflación en el conjunto de la economía:

[...] en consecuencia encontramos que en cada reino hacia el cual el dinero comienza a fluir en mayor abundancia que antes, todo adquiere una nueva cara; el trabajo y la industria ganan vida, el comerciante deviene más emprendedor, e incluso el campesino usa su arado con más entusiasmo y atención (...) Para explicar este fenómeno debemos considerar que, aunque un mayor precio de las mercaderías es la consecuencia del aumento del oro y la plata, ello no se produce inmediatamente y algún tiempo es requerido para que el dinero circule a través de todo el Estado y se haga sentir sobre las personas de todos los rangos.

La inflación ha estado presente como un recurso asociado a situaciones como el financiamiento del déficit; el crecimiento de deudas; costosas incursiones militares y reparaciones de guerras; edificación de obras suntuosas; desorden financiero; despilfarro y demás intentos de exaltación de la clase gobernante. Hülsmann (2008), apunta:

A principios de la década de 1880, los países de Occidente y sus colonias en todo el mundo adoptaron el modelo británico, bajo el que se hizo del oro un monopolio de curso legal. Esto creaba la gran ilusión de una unidad económica profunda en el mundo occidental, cuando en realidad el movimiento únicamente homogeneizaba los sistemas monetarios nacionales. La homogeneidad duró hasta 1914, cuando los bancos centrales suspendieron pagos y se prepararon para financiar la Primera Guerra Mundial mediante la imprenta (p.211). 
Aunque las tasas de inflación elevadas en países como Argentina, Brasil, México, Bolivia, entre otros, se han contenido desde la década de 2000-2010, las deudas gubernamentales se han vuelto excesivas y, por tanto, la recuperación de la economía global aún se ve lejana. Concibiendo la inflación y el crecimiento extraordinario del tamaño del gobierno como problemas serios de la sociedad, Wilhelm Ropke (1960), describe y denuncia ambos fenómenos de forma por demás elocuente:

El comunismo no constituye un peligro inmediato para los países del mundo occidental, ni alza la cabeza entre nosotros el espectro del totalitarismo, por grande que sea la amenaza de paulatina corrupción interna y de ataque inescrupuloso desde el exterior. Tampoco la economía cabalmente planificada y la socialización generalizada, ni el Estado totalitario que forzosamente las acompaña (...) las enfermedades crónicas que se extienden en secreto y por ello son más malignas (...) entre estos lentos cánceres de nuestra economía y sociedad occidentales se destacan dos: el avance, al parecer incontenible del Estado benefactor y la erosión del valor del dinero; lo que se denomina inflación reptante (p.198).

Aun cuando no se asocia a John K. Galbraith (1975), con la EA, este autor aseveró que el fenómeno de la inflación significó en el pasado ganancias extraordinarias para el naciente capitalismo norteamericano y europeo:

Los historiadores han hablado mucho, a menudo con más elocuencia que comprensión personal, de cómo los tesoros americanos financiaron, lubricaron, fomentaron o estimularon el primitivo desarrollo del capitalismo europeo (...) Los precios altos y los salarios bajos significaban grandes beneficios. Los grandes beneficios daban lugar a ahorros y eran un fuerte incentivo para la inversión (p.362).

Ludwig von Mises (1934), reconoció que la inversión o, en su caso, la 'desinversión' es el resultado de la expansión del crédito otorgado a los agentes económicos y cuyos efectos son previsibles en la perspectiva de la EA. En esta misma línea de pensamiento, De los Santos (2013), sostiene que los bancos centrales son las instituciones que propician la expansión del crédito a través de las tasas de referencia e interbancaria, respectivamente. Dicho crédito genera más gasto $\mathrm{y}$, por ende, crecimiento económico. Las malas inversiones y la distorsión inter temporal, causada por la expansión crediticia traen consigo deuda e incremento de los tipos de interés, y luego, los inevitables periodos de recesión.

De acuerdo con Bernholz (2003), países como Estados Unidos y Gran Bretaña tenían monedas estables hasta antes de la Segunda Guerra Mundial (1939-1945). Sin embargo, sus respectivos gobiernos decidieron desmantelar la estabilidad de esos regímenes monetarios con el argumento de disponer de dinero "suficiente" para encarar emergencias específicas como las crisis, guerras y demás contingencias. El patrón oro les suponía restricciones importantes, pues no podían realizar emisiones. Desde entonces, las presiones inflacionarias y otros problemas monetarios han sido constantes. Como resultado de estas emisiones monetarias el citado autor encuentra que, desde diciembre de 2007 hasta abril de 2014, la denominada masa monetaria 
de dinero en circulación y reservas bancarias se incrementaron 363,87 por ciento; ciertamente cifra muy diferente a la tasa de inflación oficial de los Estados Unidos.

Para Keynes (1973), las expansiones monetarias no representaban problema serio, pues al existir recursos ociosos se lograba reducir el desempleo y así, aumentar el ingreso. Sin embargo, Hayek (1985) sostenía que dichas expansiones del dinero y del crédito constituían distorsiones en los precios y en la asignación eficiente de recursos. De este modo, el problema seguía; no se resolvía (Cachanosky, 2008).

En la década de los veinte del siglo pasado, las ganancias de la Bolsa de Valores y del mercado inmobiliario en Estados Unidos fueron excesivas y las autoridades monetarias no le dieron la debida importancia. Según Cachanosky (1989), la expansión monetaria significó la dimensión de la Gran Depresión, por lo que el desplome ocurrido en 1929 tuvo efectos que se prolongaron hasta 1939.

En este mismo orden de ideas se manifiesta Everardo Elizondo (2015), quien sugiere que la emisión extraordinaria del dinero, en teoría, busca impulsar la economía, especialmente, a través de los valores gubernamentales y privados en el mercado, pagándose por ello con dinero ¡creado de la nada!, es decir, dinero fácil y artificial. De hecho, las crisis financieras, invariablemente son resultado de este tipo de expansiones monetarias y auges crediticios. Elizondo (2015), refiriéndose al caso mexicano de la crisis, sostiene que dicha expansión no fue casual, sino la consecuencia de una política monetaria laxa, además de acciones de rescate de mercados e instituciones en problemas.

A manera de resumen, a continuación, se presenta el Cuadro 1, en el cual los autores de la EA le confieren una significación a la inflación.

\section{Cuadro 1.}

La inflación según algunos autores de la Escuela Austriaca

\begin{tabular}{|l|l|}
\hline Autores & Concepciones respecto a la inflación \\
\hline $\begin{array}{l}\text { M. Rothbard } \\
(1990)\end{array}$ & Expansión de la oferta monetaria, más allá de la demanda agregada. \\
\hline $\begin{array}{l}\text { F. Hayek (1985) y W. } \\
\text { Ropke (1960) }\end{array}$ & $\begin{array}{l}\text { Intervención creciente del Estado benefactor para legitimarse y } \\
\text { posicionarse electoral y políticamente, lo cual requiere de crecientes } \\
\text { recursos. }\end{array}$ \\
\hline V. Mises (1934) & $\begin{array}{l}\text { Inversiones equivocadas asociadas a la expansión crediticia, provocando } \\
\text { euforia y luego crisis recurrentes (como la hipotecaría). }\end{array}$ \\
\hline $\begin{array}{l}\text { J. Huerta de Soto } \\
\text { (2009) }\end{array}$ & $\begin{array}{l}\text { Expansión crediticia artificial al otorgarse préstamos nuevos, no del } \\
\text { producto del trabajo ni del ahorro, lo cual afecta a la economía a través } \\
\text { de los precios. }\end{array}$ \\
\hline H. Hoppe (2003) & $\begin{array}{l}\text { El dinero es una mercancía que puede sustituirse y envilecerse y el } \\
\text { gobierno es un agente económico que prefiere mayores ingresos } \\
\text { (impuestos, cargos, créditos y demás) a fin de financiar su gasto } \\
\text { creciente. }\end{array}$ \\
\hline H. Hazlitt (2018) & $\begin{array}{l}\text { El aumento de los precios se relaciona con el aumento de préstamos y } \\
\text { depósitos bancarios propios de los bancos centrales. }\end{array}$ \\
\hline
\end{tabular}

Fuente: Elaboración propia con base en autores mencionados 
Respecto a las diversas experiencias de inflación en países como Estados Unidos, México, Brasil y Argentina, deben señalarse los efectos diversos en estos, pues en algunos periodos ha sido severa y extensa, además de dificultarse su cuantificación con precisión. En otros, las tasas han sido relativamente moderadas. En la Tabla 1 se observa como en el periodo 1980 a 2018, los precios fueron en general elevados en México, Brasil y Argentina, consideradas economías importantes en América Latina. Aunque los datos no se muestran aquí, entre 19731974 y luego entre 1979 y 1980, se produjo la primera y segunda crisis petrolera a nivel internacional. Los precios del crudo se incrementaron considerablemente. Estados Unidos experimentó niveles elevados en este indicador entre 1980 y 1981, pero después se observa una reducción a lo largo del periodo.

No fueron así las experiencias de los demás países (Tabla 1), pues se observa un patrón de elevación de precios, especialmente entre los 80 y parte de los 90 . Del 2000 a la fecha se advierte la existencia de políticas en lo general más efectivas contra la inflación. Debe señalarse el que los bancos centrales han recurrido a medidas como las metas de inflación anual y se ha determinado su autonomía respecto al gobierno. También se ha puesto mayor atención al comportamiento de los precios no solo de manera interna, pues también organismos financieros internacionales, bancos, corporaciones y demás agentes económicos están atentos al comportamiento de dicha variable.

De la Tabla 1 se desprende que, a lo largo del periodo, en promedio, la inflación en Estados Unidos fue 2.99 por ciento, la de México 25.17, la de Brasil 309.12 y la de Argentina 219.99. Los elevados niveles de esta variable ocurrieron entre 1989-1990 y luego entre 19931994. Según se advierte fueron particularmente adversos en Argentina y Brasil. En menor medida se observó un incremento en México en los años en que ocurrieron las devaluaciones del peso $(1982,1987,1988$ y 1994) Los precios muy elevados suponen enormes dificultades para que el grueso de la población de estos países accediese a los bienes y servicios esenciales dado el poder de compra erosionado. El problema de la inflación es estructural y recurrente entre algunos países de América Latina (en este caso es más grave en Argentina y Brasil que en México, aunque en Estados Unidos aún menor). La estadística descriptiva de la Tabla 1 proporciona elementos para observar a mayor detalle las disparidades en esta variable.

Tabla 1.

Tasas de inflación en Estados Unidos, México, Brasil y Argentina (1980-2018)

\begin{tabular}{|r|r|r|r|r|r|}
\hline \multicolumn{1}{|c|}{ Periodo } & \multicolumn{1}{|c|}{ Estados Unidos } & \multicolumn{1}{|c|}{ México } & \multicolumn{1}{c|}{ Brasil } & \multicolumn{2}{|c|}{ Argentina * } \\
\hline 1980 & 13.55 & 26.35 & nd & 100.8 & 104.5 \\
\hline 1981 & 10.33 & 27.93 & 101.73 & 164.8 \\
\hline 1982 & 6.13 & 58.91 & 100.54 & 343.8 \\
\hline 1983 & 3.21 & 101.87 & 135.03 & 626.7 \\
\hline 1984 & 4.30 & 65.45 & 192.12 & 672.2 \\
\hline 1985 & 3.55 & 57.75 & 225.99 & 90.1 \\
\hline 1986 & 1.90 & 86.23 & 147.14 & 31.3 \\
\hline 1987 & 3.66 & 131.83 & 228.34 & 343 \\
\hline 1988 & 4.08 & 114.16 & 629.11 & 3079.8 \\
\hline 1989 & 4.83 & 20.01 & $1,430.73$ & \\
\hline
\end{tabular}




\begin{tabular}{|c|c|c|c|c|}
\hline 1990 & 5.40 & 26.65 & $2,947.73$ & 2314 \\
\hline 1991 & 4.23 & 22.66 & 432.79 & 171.7 \\
\hline 1992 & 3.03 & 15.51 & 951.96 & 24.9 \\
\hline 1993 & 2.95 & 9.75 & $1,927.38$ & 10.6 \\
\hline 1994 & 2.61 & 6.97 & $2,075.89$ & 4.2 \\
\hline 1995 & 2.81 & 35.00 & 66.01 & 3.4 \\
\hline 1996 & 2.93 & 34.38 & 15.76 & 0.2 \\
\hline 1997 & 2.34 & 20.63 & 6.93 & 0.5 \\
\hline 1998 & 1.55 & 15.93 & 3.20 & 0.9 \\
\hline 1999 & 2.19 & 16.59 & 4.86 & -1.2 \\
\hline 2000 & 3.38 & 9.49 & 7.04 & -0.9 \\
\hline 2001 & 2.83 & 6.37 & 6.84 & -1.1 \\
\hline 2002 & 1.59 & 5.03 & 8.45 & 25.9 \\
\hline 2003 & 2.27 & 4.55 & 14.71 & 13.4 \\
\hline 2004 & 2.68 & 4.69 & 6.60 & $\overline{4.4}$ \\
\hline 2005 & 3.39 & 3.99 & 6.87 & 9.6 \\
\hline 2006 & 3.23 & 3.63 & 4.18 & 10.9 \\
\hline 2007 & 2.85 & 3.97 & 3.64 & 8.8 \\
\hline 2008 & 3.84 & 5.12 & 5.68 & 8.6 \\
\hline 2009 & -0.36 & 5.30 & 4.89 & 6.3 \\
\hline 2010 & 1.64 & 4.16 & 5.04 & 10.8 \\
\hline 2011 & 3.16 & 3.41 & 6.64 & 9.5 \\
\hline 2012 & 2.07 & 4.11 & 5.40 & 10 \\
\hline 2013 & 1.46 & 3.81 & 6.20 & 10.6 \\
\hline 2014 & 1.62 & 4.02 & 6.33 & 10 \\
\hline 2015 & 0.12 & 2.72 & 9.03 & 20.8 \\
\hline 2016 & 1.26 & 2.82 & 8.74 & 46.8 \\
\hline 2017 & 2.13 & 6.04 & 3.45 & 26.9 \\
\hline 2018 & 2.44 & 4.90 & 3.66 & 42.8 \\
\hline Promedio & 2.99 & 25.17 & 309.12 & 219.99 \\
\hline Máximo & 10.33 & 131.83 & $2,947.73$ & $3,079.80$ \\
\hline Mínimo & $(0.36)$ & 2.72 & 3.20 & $(1.20)$ \\
\hline Desviación Estándar & 1.78 & 33.63 & 674.33 & 619.11 \\
\hline
\end{tabular}

Fuente: Elaborado por el autor con base en estadísticas del Banco Mundial.

*Banco Central de la República Argentina (2019).

$\mathrm{Nd}$. No disponible

Los bancos centrales son instituciones creadas con el propósito de contribuir a la estabilización de precios y al crecimiento de la economía. Tienen ante sí enormes desafíos en el mundo cambiante actual. La creación de estas instituciones se justifica en la medida en que se cumplen los objetivos de velar por una moneda estable. La Gráfica 1 muestra el crecimiento de la oferta de dinero (M1), la cual se entiende como la forma del dinero más liquida que contempla el efectivo en manos de la gente, además del dinero depositado en cuentas bancarias. Entre los países seleccionados se advierte que, como rasgo común, todos ellos experimentan una etapa de estabilidad relativa hasta mediados de los 90. Luego, ocurre la expansión, especialmente en el contexto de la Gran Recesión de 2007-2009. La expansión del 
dinero (y del crédito), es con mucho mayor al de la producción y el ahorro internos. La expansión de la oferta del dinero ha tendido a incrementarse aun cuando existen metas de inflación, bancos centrales autónomos, leyes monetarias y demás medidas.

Gráfica 1. Oferta Monetaria (M1) de México, Japón, Estados Unidos y Brasil

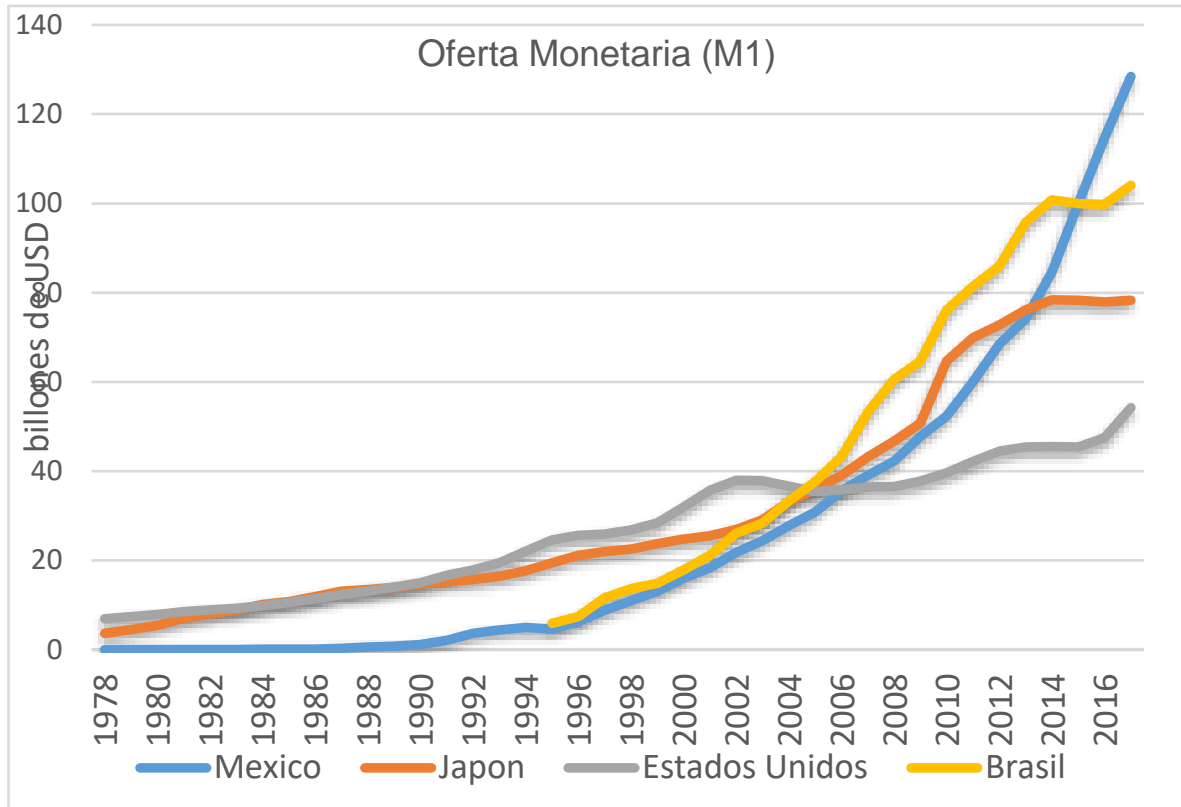

Fuente: Organización para la Cooperación y el Desarrollo Económico (2018). Estadísticas de oferta monetaria (M1).

Asociado a la inflación se halla el déficit público. Este puede ser causado por factores como la baja recaudación, la pérdida de dinamismo económico y el costo creciente del servicio de la deuda. Con déficit, los agentes económicos tienen un entorno complicado y el margen de maniobra del gobierno se reduce. Existen presiones para mantener y aun expandir el nivel de gasto: el tamaño del gobierno, el gasto militar, el Estado de bienestar, además de inversiones en infraestructura indispensables para atraer la inversión y el desarrollo social. La Gráfica 2 muestra el crecimiento de la deuda pública como porcentaje del Producto Interno Bruto (PIB) entre Grecia, Italia, Argentina, España, Japón, Estados Unidos y México. En todos ellos, en casi medio siglo creció varias veces su tamaño: en Grecia poco más de siete veces, en Italia y Argentina poco más de cuatro veces, en Estados Unidos y en México poco más de tres veces. 
Gráfica 2. Deuda como porcentaje del Producto Interno Bruto (1970-2019) entre Grecia, Italia, Argentina, España, Japón, Estados Unidos y México

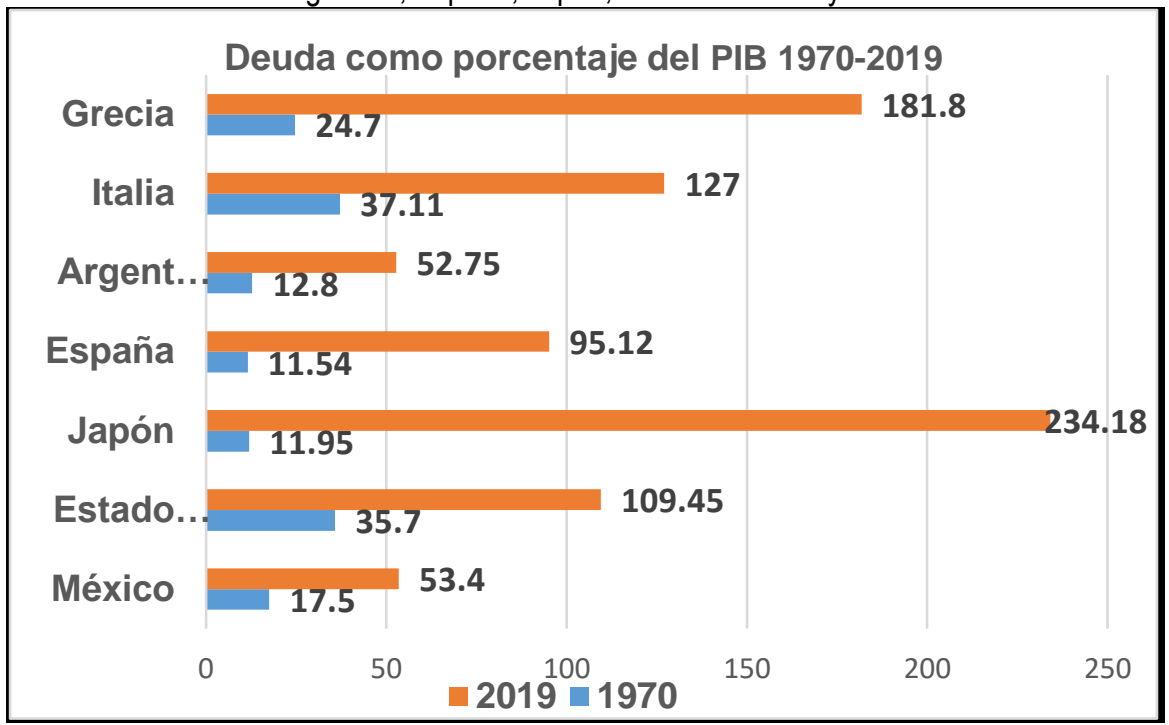

Fuente: International Fund Monetary (2019).

\section{La inflación según las diversas escuelas del pensamiento económico contemporáneo}

En este apartado se describe de forma concisa lo que sostienen las principales escuelas de pensamiento económico con respecto al fenómeno de la inflación.

La escuela estructuralista. Esta tiene su origen en Latinoamérica y sostiene que la inflación no es solamente el resultado de los empujes de los costos, del impulso de la demanda o del gasto público. El problema reside en su estructura económica y política proclive para favorecer el crecimiento continuo de los precios dado sus déficits persistentes. Las economías de los países latinoamericanos son vulnerables a la inflación, el desempleo y demás problemas económicos estructurales. Sunkel (1989), agrega que la insuficiencia en el ahorro y la desigual distribución del ingreso son adversas para el crecimiento económico. El comercio internacional no siempre les resulta ventajoso porque la capacidad de exportación se ve limitada debido a la falta de diversificación de bienes, con lo cual se hace inestable su desarrollo.

La escuela neoestructuralista. Esta corriente otorga importancia al corto y al largo plazo en los equilibrios macroeconómicos. En ese sentido, resulta fundamental reducir el tamaño del déficit a fin de establecer precios moderados. De acuerdo con Berthomieu, Ehrart y Hernández-Bielma (2006), los factores que afectan la inflación se asocian con los contratos de indexación que conducen a una inercia de propagación a los demás agentes. Dicha escuela reconoce la necesidad de aplicar medidas antiinflacionarias de manera gradual (p.5-6).

La escuela monetarista. De acuerdo con esta escuela, la inflación es el resultado de operar con fuentes escasas de recursos para cubrir las necesidades. Por lo tanto, se recurre al aumento de la cantidad de dinero en circulación, de modo que tanto las empresas privadas como el mismo gobierno financian su expansión. En la realidad, no hay crecimiento tal que no venga 
acompañado del crecimiento en los precios, aunque estos sean mínimos. Según Friedman (1982), la inflación se puede evitar si se decide no incrementar los créditos o la cantidad de dinero para impulsar el crecimiento.

La escuela keynesiana. De acuerdo con esta corriente, las expansiones de la demanda agregada se asocian al fenómeno inflacionista, especialmente cuando la economía se encuentra lejos del pleno empleo. La inflación se explica por la conjunción de tres factores: demanda, costos e inercia. Según Keynes (1973), ante la insuficiencia de ingresos fiscales, los gobiernos recurren a la creación de dinero para "cubrir el déficit". Así, este problema debe verse como un impuesto disfrazado que daña a la economía real. La forma de enfrentar el problema monetario en cuestión se relaciona con la magnitud de la disparidad de la renta y el consumo, reduciéndose así el volumen de la inversión (Sierra Lara, 2007).

La Escuela Austriaca. En la perspectiva de esta, la inflación se relaciona con el incremento de la oferta monetaria. El fenómeno se entiende a partir de la distribución de precios mayores que los proporcionales a los de los ingresos de la población. Como solución, se propone la eliminación del monopolio emisor de moneda, en este caso, los bancos centrales. Plantea, además, que la moneda de curso legal se respalde por activos tangibles de mayor certidumbre como el oro, la plata y el platino. Para Ludwig von Mises (1978), la inflación no debe ser considerada un fenómeno aislado: más bien se circunscribe en el marco de acciones de carácter político, ideológico y económico. Para esta escuela de pensamiento, la inflación debe ser concebida como una parte inherente del imperialismo, el militarismo, el proteccionismo, el estatismo y el socialismo (p.9).

\section{Efectos inflacionarios sobre los agentes económicos}

La ciencia económica contemporánea sigue con cuidado la trascendencia y gravedad de las crisis de carácter monetario. De hecho, no hay forma de que los hechos sociales y económicos pueden aislarse del contexto complejo en que se hallan. Existe una grave afectación del nivel de precios respecto al empleo, la producción y demás variables macroeconómicas.

En el orden macroeconómico, la inflación aumenta cuando la producción y el empleo son elevados, especialmente en el corto plazo. En el orden microeconómico, cuando la inflación es muy elevada se distorsionan los precios de los factores de producción. El tipo de interés real es afectado, por lo que el público suele deshacerse del dinero, adquiriendo bienes lo más pronto posible.

Con la inflación elevada se incrementan considerablemente los precios de los bienes raíces, automóviles nuevos y demás bienes. Si los costos de los intereses son menores que los beneficios obtenidos, invertir en un bien es una opción preferida. El medio financiero, consciente de que el problema es grave, ha incorporado instrumentos que prevén cambios en los tipos de interés por los préstamos y créditos otorgados. De igual modo, los sindicatos de trabajadores buscan compensar la pérdida del valor del dinero revisando los salarios, anticipándose con ello _ inflación esperada - o o bien compensando las pérdidas de los salarios reales atribuidas al mismo fenómeno en cuestión (Elizondo, 2001).

En este contexto, debe señalarse el hecho de que el aumento de precios no es uniforme ni neutral, sino que tiende a modificar la estructura económica en cuanto al tipo de interés, consumo e inversión, produciéndose así ganadores y perdedores (efecto Cantillon). Ello justamente se advirtió en el contexto de la Gran Recesión (2007-2009), pues a pesar de las 
enormes emisiones de dinero realizadas por los bancos centrales y los rescates gubernamentales, estos recursos no llegaron a la población más pobre de los países afectados. El poder de compra de estos ciudadanos se vio considerablemente erosionado.

Existen, adicionalmente, efectos sobre el gobierno mismo, pues con altos niveles de precios es difícil determinar con precisión el presupuesto público. Cuando los impuestos son progresivos, la inflación eleva los tipos impositivos de los contribuyentes, con lo cual se recauda más impuestos sin modificar la legislación. Al decir de Ampudia Márquez (2007), "otro efecto de la inflación es el del tipo de cambio" (p. 63). En los países proclives a elevados precios, los bancos centrales utilizan el tipo de cambio como ancla antiinflacionaria, empleando diversos regímenes cambiarios: tipo de cambio fijo, dolarización, caja de conversión, control cambiario, devaluaciones programadas y tipo de cambio intervenido. En este contexto, no pueden quedar fuera los empresarios. Este sector suele pugnar para que las leyes les permitan depreciar con mayor rapidez y a un costo de reposición.

Pero ¿quiénes ganan entonces en periodos de inflación? Naturalmente, aquellos que han recurrido al endeudamiento a sabiendas de que con tipos de intereses bajos van a pagar después dinero barato. También las empresas grandes que disponen de activos hipotecan a gran valor. Como medida precautoria las empresas elaboran listas de precios con la leyenda "precios sujetos a cambio sin previo aviso". La dolarización de los activos en venta constituye un mecanismo de protección contra la pérdida del valor de la moneda nacional.

\section{Políticas de estabilización}

Cuando los gobiernos aplican medidas correctivas, lo hacen a sabiendas de la existencia de costos sociales y aun políticos en que incurren. Algunas acciones gubernamentales para lidiar con este problema tienen denominaciones como: programa de reordenación económica, saneamiento financiero, pacto económico, políticas monetarias y tributarias estabilizadoras, entre otras; todas ellas dirigidas a establecer controles sobre precios y salarios. Adoptar este tipo de políticas no es un asunto sencillo, pues se requiere trabajo de consenso político y de adecuación a los mercados financiero, exterior, industrial y laboral, a fin de reducir la incertidumbre respecto a la eficacia del programa de estabilización. Al adoptar este tipo de políticas, los gobiernos deben considerar factores cruciales como la magnitud de la crisis, la posición de las políticas económicas, la coyuntura social, así como a los intereses internacionales.

Básicamente, se reconocen dos estrategias alternativas para resolver el problema en cuestión. La primera de ellas es la 'estrategia de gradualismo'. Esta política reconoce la magnitud de la problemática, pero es suave, lenta y pragmática para con la reducción de la producción y el empleo. No lleva prisa, pues reconoce los riesgos de brotes de inconformidad social y agitación política. El objetivo subyacente es que la inflación gradualmente se vaya reduciendo, afectando lo menos posible a la producción potencial. Los bancos centrales la adoptan en países con tasas moderadas. La segunda se denomina 'estrategia de choque'. Esta pretende reducir la tasa de inflación lo más rápido posible, sin importar mucho los efectos.

La anterior estrategia pretende reducir el crecimiento del dinero y, por ende, de la producción en el corto plazo. Puede ser que el gobierno se vea muy presionado a actuar desde dentro — por factores políticos, empresariales 0 sociales —, o desde fuera — por organismos internacionales, acreedores, bancos centrales y demás-. Esta estrategia reduce el choque de 
la tasa de crecimiento del dinero, aunque ello genere un entorno recesivo y un elevado desempleo en el corto plazo.

Las políticas de estabilización implican elegir una senda óptima de inflación y de empleo. La senda más rápida reduce el desempleo rápidamente, pero lo hace sin conseguir grandes avances en el problema en cuestión. Según el modelo de relación de la inflación y el desempleo para reducir la inflación rápidamente, denominado la 'curva de Phillips', el desempleo debe ser elevado y la recuperación lenta (Blanchard, 2017).

En este mismo orden de ideas, González Huerta (2004), considera que "las políticas de estabilización no necesariamente están comprometidas con objetivos distintos al de mantener bajo control los precios\# (p. 16). Lo aduce en el sentido de que la política monetaria y fiscal restrictivas, además de la política de apreciación cambiaria, ciertamente reducen la inflación, pero a costa de atentar sobre la competitividad de la producción nacional como sobre el mercado interno, lo que afecta los beneficios de las empresas y a la generación de empleos.

Así, pues, en este contexto es válido el razonamiento de que la producción, los salarios y los precios son variables indisolubles. A medida en que un país se encuentre cerca del umbral de una recuperación, más cerca se halla de incurrir en niveles más altos de precios. De este modo, el pleno empleo de los factores productivos tiene relación inversamente proporcional con los precios.

La realidad de este fenómeno ofrece lecciones que deben de considerarse seriamente. En cuanto a los riesgos potenciales asociados a la inflación como los altos niveles de deuda pública y privada, Mueller (2012), señala la dificultad que enfrentan las instituciones referidas para elevar los tipos de interés, dado su impacto directo en la inflación. Esto atañe a la política monetaria y, en este contexto, bajar dichas tasas no es suficiente, pues no se acompaña de otros elementos de política económica esenciales. De allí, la receta de inyectar liquidez y mantener al mínimo posible la inflación ha dejado de funcionar y de ser efectiva (p.1-2).

Por tanto, en una gran cantidad de países, entre ellos México, el crecimiento económico es muy modesto, lo cual no es un asunto menor. Alejandro Nadal (2012), manifiesta su preocupación destacando que la presión inflacionaria ha sido más o menos dominada, aunque ello a costa de sacrificios como la contención salarial, la sobrevaluación cambiaria y las posturas restrictivas en materia fiscal y monetaria. Desde su punto de vista, lo anterior ha sido errático y lo más grave es que los gobiernos se han ufanado de la supuesta estabilidad macroeconómica. Lo peor es el estancamiento y la pobreza generalizada (p.1).

\section{Conclusiones}

El presente trabajo examina el problema de la inflación según la perspectiva de la Escuela Austriaca. Se ha realizado una revisión de la literatura existente. Esta propuesta se ha contrastado con la corriente dominante o mainstream, destacándose el origen del problema en mención, asimismo, se han señalado los efectos negativos. También se han señalado experiencias respecto al problema en cuestión en países como Estados Unidos, México, Brasil y Argentina, evidenciándose cuan grave ha sido. Adicionalmente, se ha mostrado con datos históricos, como han crecido las emisiones de dinero y de la deuda pública entre algunos países, con lo cual se ha agravado el problema.

El trabajo también describe de manera concisa lo que sostienen algunos autores de la EA respecto a la inflación, contrastándola con las escuelas estructuralistas, neo estructuralista, 
monetaria y keynesiana. También se ha señalado los efectos que tiene la inflación sobre los agentes económicos, siendo estos más gravosos en las familias de ingresos bajos. Las características de las políticas de estabilización es un apartado que también se incluye, pues se trata de medidas que los gobiernos adoptan para contener el problema económico en cuestión, diferenciándose las estrategias gradualista y de choque.

Según la EA, los factores detonantes de la inflación lo constituyen, en primer término, las operaciones en el mercado abierto, en las cuales el gobierno compra títulos de entidades particulares en las bolsas de comercio, pagando con dinero sin respaldo. Lo mismo se hace con las emisiones de títulos. En segundo lugar, se considera como factor interviniente la manipulación de las tasas de interés. En este caso, se facilita crédito barato al público, reduciendo el máximo dichas tasas de préstamos. Con esta reducción artificial el ahorrador retira su dinero, en tanto que las solicitudes de préstamos se expanden. En tercer lugar, se enlista el déficit financiero del presupuesto: de no haber inflación no se produce el déficit. Este último es impulsado por la emisión de dinero sin respaldo. Al recurrir a este, el gobierno reduce sus propias reservas e incurre en altos costos de oportunidad. En ese sentido, bajar gastos y elevar los impuestos constituye una medida políticamente incorrecta, más aún cuando hay en ciernes contiendas electorales.

Aun cuando no se ha señalado en este trabajo, existen países que están enfrentando problemas graves de tipo económico y social asociados a elevados niveles de inflación, tipos de cambio adversos, desabasto de bienes, con lo cual la población se encuentra desesperada. Venezuela es el caso más emblemático de América Latina, pero estos problemas se experimentan, con diferentes matices, también en algunos países de Centro América y de otros lugares del mundo. La economía para que sirva a la sociedad debe promover la participación de los agentes políticos, empresariales y de la sociedad civil. El camino de elevar la producción y el empleo se relaciona positivamente con la estabilidad de precios.

La evidencia es clara, pues el mayor peso de la inflación históricamente ha recaído en los grupos de bajos ingresos. Los empresarios que ganan son aquellos que invierten en bienes raíces, equipo, inventarios, manipulan las deudas, especulan con otras monedas y realizan otras acciones preventivas. En algunos casos, la inflación ha significado ganancias extraordinarias para las grandes empresas. Debe aquí mismo señalarse que los precios elevados pueden ser, adicionalmente, derivados de perspectivas de escasez de bienes y de especulación. La estructura de mercado con alta concentración debido a la existencia de monopolios y oligopolios es proclive a la elevación de los precios. Por si fuera poco, en casos particulares de contingencia los gobiernos elevan impuestos y precios de bienes y servicios. La mala distribución del ingreso es una consecuencia arbitraria; entre más fuerte sea, tendrá efectos diferenciados entre los sectores de la población.

Con base a lo que se ha expresado hasta aquí, se debe replantear el papel que juega la política monetaria respecto al control de inflación, pues los bancos centrales no han impedido las crisis monetarias ocurridas hasta ahora. Es preciso recordar que las diversas experiencias de controles de los tipos de cambio, de interés y de precios no han sido capaces de erradicar la inestabilidad y riesgos constantes. Las alternativas adoptadas por los gobiernos y sus respectivos bancos centrales han sido los consejos monetarios, regímenes de libertad bancaria y aun la misma propuesta de la EA de suprimir los bancos centrales, la cual en principio se antoja osada. 
En una época como la nuestra, en que la ciencia económica ha progresado notoriamente, debieran diagnosticarse a tiempo las grandes problemáticas macroeconómicas y así prescribir las medidas de ajuste a fin de evitar la profundización y la extensión de las crisis. La experiencia de diversos países a través del siglo XX y lo que va del XXI demuestra que el crecimiento y el bienestar de la población son muy limitados en aquellas naciones con elevada inflación. En ese sentido, el objetivo de mantener baja la inflación no es suficiente si no se procura la estabilidad monetaria y la expansión prudente del crédito, además de otras metas sostenibles.

La era de la discontinuidad económica ha dejado atrás los moderados cambios en los ciclos. Tasas menores de inflación y de desempleo eran alicientes para la inversión con relativa certidumbre. Sin embargo, esos años quedaron atrás desde mitad de los setenta del siglo XX. Han sido sustituidos por factores como un entorno con riesgos e incertidumbre mayores, menor participación del Estado en la economía, crecientes niveles de deuda, bajo nivel de crecimiento económico y de un reciente repliegue hacia el proteccionismo comercial. Las experiencias de estas décadas agitadas debieran arrojar más luz respecto a cómo evitar que la inflación y los demás problemas económicos agobien a la sociedad a fin de salir mejor librados de los desafíos de la economía abierta. Aún más que esto último: la cuestión por resolver será cómo prevenir la fuerte exposición a la volatilidad financiera, connatural a los tipos de cambio, y a los demás problemas económicos que afectan a la sociedad actual.

\section{Referencias Bibliográficas}

Ampudia Márquez, Nora (2007). El efecto del traspaso de la inflación, debilidades de la política monetaria e impacto en el mercado laboral, en Macroeconomía del crecimiento sostenido (primera edición). Editorial Porrúa. México.

Banco Central de la República Argentina (2019). Estadísticas de inflación. Argentina.

Banco Mundial (2019). Estadísticas de inflación para diferentes países. Extraído de: https://datos.bancomundial.org/indicador/FP.CPI.TOTL.ZG?locations=BR\&view=chart Consulta 03/12/2019.

Bernholz, Peter (2003). Monetary regimes and inflation: history economic, and political relationship. Eduard Elgar. Chelttenham, U.K. Doi: 10.2202/1145-6396.1120

Berthomieu, Claude; Ehrhart, Christophe y Hernández-Bielma, Leticia (2006). Estabilización, ajuste externo y estrategia de desarrollo: El neo estructuralismo como alternativa al neoliberalismo. Revista de Análisis Económico. Volumen XXI, número 48. México (Pp.5-30).

Blanchard, Olivier (2017). Macroeconomía (séptima edición). Prentice Hall. México.

Borgucci, Emmanuel (2011). Teoría Austriaca de los ciclos económicos y la economía venezolana. Revista Telos. Volumen 13. No.2. Venezuela (Pp. 175-193). Extraído de: https://www.researchgate.net/publication/277269318_Teoria_austriaca_de_los_ciclos economicos y la economia venezolana Consulta: 02/05/2018.

Cachanosky, Juan C. (1989). La crisis de los treinta. Revista Libertas 1. Buenos Aires (Pp.1-33).

Extraído de: http://www.eseade.edu.ar/files/Libertas/40 7 Cachanosky.pdf Consulta: $11 / 08 / 2018$. 
Cachanosky, Juan C. (2008). La Escuela Austriaca de economía. Revista de Instituciones, Ideas y Mercados. Buenos Aires. Extraído de:https://www.eseade.edu.ar/wpcontent/uploads/2016/08/49_2_cachanosky.pdf. Consulta: 02/05/2018.

De los Santos, Pablo (2013). La trampa del crédito. Reforma. México.

Elizondo, Everardo (2001). ¿Se Acabó la inflación?: No todavía. Reforma. México.

Elizondo, Everardo (2015). La ilusión de la alquimia. Reforma. México.

Friedman, Milton (1982). Paro e inflación (primera edición). Unión Editorial. España.

Galbraith, John Kenneth. (1975). El Dinero (primera edición). Biblioteca de Economía. España. González Huerta, Arturo (2004). Política antiinflacionaria y estancamiento, revista Economía Informa, número 326. México

Hayek, Friedrich (1985). La desnacionalización del dinero (primera edición). Ediciones Orbis. España.

Hayek, Friedrich (1996). Precios y producción. Una explicación de las grandes crisis de las economistas capitalistas del siglo XX. Ediciones Aosta. España.

Hazlitt, Henry (2018). Inflación para principiantes. Mises Institute. Extraído de: https://mises.org/es/wire/inflation-para-principiantes Consulta: 11/17/2018.

Hoppe, Hans H. (2003). Government, Money and international Politics. Ethics \& Politics. Extraído de: https://www2.units.it/etica/2003_2/hoppe.pdf Consulta: 22/09/2019.

Huerta de Soto, Jesus (2009). Dinero, crédito bancario y ciclos económicos (cuarta edición). Unión Editorial. España.

Hume, David (1750). Of Money: Essays. Oxford University Press. Oxford.

Hülsmann, Guido (2008). The Ethics of money production. Von Misses Institute. Auburn. Extraído de: https://mises.org/library/ethics-money-production Consulta: 02/03/2018.

International Fund Monetary (2019). Debt as percentage of GDP. Extraído de: https://www.imf.org/external/datamapper/DEBT1@DEBT Consulta: 18/06/2019.

Johnson, Harry (1985). Inflación, revolución y contra revolución keynesiana y monetarista. Ediciones Orbis. España.

Keynes, John Maynard (1973). The General theory of employment, interest and money. London Macmillan Co. United Kingdom.

Mises, Ludwig von (1934). La Teoría del dinero y el crédito. Unión Editorial. España.

Mises, Ludwig von (1978). On the manipulation of money and credit: Three treatise on trade cycle. Liberty Fund. United States.

Mueller, P. Antony (2012). Inflation targeting hits the wall, Mises Institute Extraído de: https://mises.org/library/inflation-targeting-hits-wall Consulta: 19/07/2019

Nadal, Alejandro (2012). Estabilidad macroeconómica: ¿déficit cero?, La Jornada. México. Extraído de: https://www.jornada.com.mx/2012/12/12/opinion/028a1eco Consulta: 13/09/2019.

Organización para la Cooperación y el Desarrollo Económico (2018). Extraído de: https://data.oecd.org/money/narrow-money-m1.htm Consulta:18/06/2019.

Perdomo Ruiz, Harold (2017). La inflación de la Escuela Austriaca. Las implicaciones de su uso en la política económica. No. 108. Revista Econógrafos. Colombia. Extraído de: http://www.fce.unal.edu.co/publicaciones/images/econografos/documntoseconografos-economia-108.pdf Consulta: 09/06/2018.

Ropke, Wilhelm. (1960). A humane Economy. Henry Regnery Co. United States. 
Rothbard, Murray. N. (1990). La Teoría austriaca del dinero. Revista Libertas. Número 13, Argentina. (Pp.1-19).

Samuelson, Paul y Nordhaus, William (2010). Macroeconomía (quinceava edición). McGraw Hill. México.

Sierra Lara, Yoandris (2007). El Pensamiento económico de John M. Keynes y Milton Friedman: Un estudio de sus teorías a través de cuatro problemas centrales. Extraído de: https://www.researchgate.net/publication/5016202_El Pensamiento_Economico de John_Maynard_Keynes_y_Milton_Friedman_Un_estudio_de_sus_teorias_a traves de 4 _problemas centrales/download. Consulta: 02/25/2018.

Smith, Adam (1983). La Riqueza de las naciones I (primera edición). Ediciones Orbis. España. Sunkel, Osvaldo (1989). Institucionalismo y estructuralismo. Revista de la CEPAL. Santiago de Chile (Pp. 147-156). Extraído de: https://repositorio.cepal.org/bitstream/handle/11362/11743/038121132_es.pdf?seque nce=1\&isAllowed=y Consulta: 22/06/2019. 